\title{
Absence of Fungal Spore Internalization by Bronchial Epithelium in Mouse Models Evidenced by a New Bioimaging Approach and Transmission Electronic Microscopy
}

\author{
Blandine Rammaert, ${ }^{* \dagger \ddagger \S}$ Grégory Jouvion, ${ }^{9 \|}$ Fabrice de Chaumont, ${ }^{* *}$ Dea Garcia-Hermoso, ${ }^{* \dagger}$ Claire Szczepaniak, ${ }^{\dagger \dagger}$ \\ Charlotte Renaudat, ${ }^{* \dagger}$ Jean-Christophe Olivo-Marin, ${ }^{* *}$ Fabrice Chrétien, ${ }^{\Phi \|}{ }^{\star \ddagger}$ Françoise Dromer, ${ }^{\dagger}$ and Stéphane Bretagne ${ }^{\star \S, \delta \S}$
}

\begin{abstract}
From the Units of Molecular Mycology, *Human and Animal Model Histopathology," and Biological Image Analysis, ** Institut Pasteur, Paris; the URA $3012,{ }^{\dagger}$ The French National Centre for Scientific Research (CNRS), Paris; the Infectious and Tropical Diseases Service, ${ }^{\ddagger}$ Hôpital Necker-Enfants Malades, APHP, Paris; Paris Diderot University, ${ }^{\S}$ Bio Sorbonne Paris Cité, Paris; Paris Descartes University, " PRES Sorbonne-Paris-Cité, Paris; the Cellular Health Imaging Center ${ }^{\dagger \dagger}$ Université d'Auvergne, Clermont Ferrand; the Neuropathology Service ${ }^{\ddagger \ddagger}$ Centre Hospitalier Sainte-Anne, Assistance Publique-Hôpitaux de Paris, Paris; and the Parasitology-Mycology Service, ${ }^{\S \subseteq}$ Hôpital St Louis, AP-HP, Paris, France
\end{abstract}

\author{
Accepted for publication \\ April 27, 2015. \\ Address correspondence to \\ Blandine Rammaert, M.D., \\ Ph.D., Unité de Mycologie \\ Moléculaire, Institut Pasteur, 28 \\ rue du Dr. Roux, 75015 Paris, \\ France. E-mail: blandine.r- \\ ammaert@pasteur.fr or \\ brammaert@yahoo.fr.
}

\begin{abstract}
Clinical data and experimental studies suggest that bronchial epithelium could serve as a portal of entry for invasive fungal infections. We therefore analyzed the interactions between molds and the bronchial/ bronchiolar epithelium at the early steps after inhalation. We developed invasive aspergillosis (Aspergillus fumigatus) and mucormycosis (Lichtheimia corymbifera) murine models that mimic the main clinical risk factors for these infections. Histopathology studies were completed with a specific computer-assisted morphometric method to quantify bronchial and alveolar spores and with transmission electron microscopy. Morphometric analysis revealed a higher number of bronchial/bronchiolar spores for $A$. fumigatus than L. corymbifera. The bronchial/bronchiolar spores decreased between 1 and 18 hours after inoculation for both fungi, except in corticosteroid-treated mice infected with A. fumigatus, suggesting an effect of cortisone on bronchial spore clearance. No increase in the number of spores of any species was observed over time at the basal pole of the epithelium, suggesting the lack of transepithelial crossing. Transmission electron microscopy did not show spore internalization by bronchial epithelial cells. Instead, spores were phagocytized by mononuclear cells on the apical pole of epithelial cells. Early epithelial internalization of fungal spores in vivo cannot explain the bronchial/bronchiolar epithelium invasion observed in some invasive mold infections. The bioimaging approach provides a useful means to accurately enumerate and localize the fungal spores in the pulmonary tissues. (Am J Pathol 2015, 185: 2421-2430; $h$ ttp://dx.doi.org/10.1016/j.ajpath.2015.04.027)
\end{abstract}

Invasive pulmonary mold infections dominated by invasive aspergillosis and mucormycosis are a major threat for immunocompromised patients with a 3-month overall mortality rate $>45 \%$ and an increasing incidence. ${ }^{1-5}$ Among the different clinical presentations of invasive aspergillosis, the broncho-invasive form is characterized by bronchial and peribronchial hyphal invasion and is reported in $14 \%$ to $38 \%$ of patients receiving corticosteroids. ${ }^{6-8}$ Mucormycosis can also involve bronchi, either trachea and main bronchi especially in diabetic patients, or small bronchi/bronchioles as suggested by the presence of tree-in-bud opacities on chest tomodensitometry. ${ }^{9,10}$ Altogether these data suggest that bronchial/bronchiolar epithelium could serve as a portal of entry for Aspergillus spp. and Mucorales.

Several not exclusive hypotheses for the bronchial invasion by fungi can be raised and were mostly assessed

\footnotetext{
Supported by the Prix de la Chancellerie des Universités de Paris, Legs Poix and the Institut Pasteur (Program de Recherche transversal, PTR 409). B.R. is recipient of Institut Pasteur, Ecole Polytechnique, Arts et Metiers ParisTech, École supérieure de physique et de chimie industrielles de la ville de Paris (ESPCI Paris Tech), Université de technologie de Compiégne (UTC), Commissariat á l'énergie atomique et aux énergies alternatives (CEA), CNRS, Labex BioPsy, and AP-HP fellowships.

Disclosures: None declared.
} 
through in vitro models: i) internalization of fungal spores by host cells (human bronchial epithelial cell lines, and mammal primary tracheal or bronchial epithelial cells ${ }^{11-13}$ ); ii) chemical disruption of the epithelial-cell barrier by production of fungal proteases or toxins leading to the cleavage of tight junctions ${ }^{13-15}$; iii) interactions with professional phagocytic cells such as dendritic cells that could bypass the epithelial barrier ${ }^{16}$; and iv) damage of bronchial epithelial cells. ${ }^{13,17}$

We therefore studied the early events after spore inhalation, knowing that most of the pathogenic fungi can be internalized by nonprofessional phagocytic cells. ${ }^{18}$ Aspergillus fumigatus was chosen as the leading cause of invasive aspergillosis and by far the most studied mold in the context of invasive fungal disease. We added for comparison Lichtheimia corymbifera (formerly Absidia corymbifera) which ranks second or third as a cause of mucormycosis. ${ }^{4,5}$ Both molds share common features such as a ubiquitous presence in the environment, a spore size $<3 \mu \mathrm{m}$ in diameter, the ability to grow at $>37^{\circ} \mathrm{C}$, and the main risk factors (neutropenia and corticosteroid) predisposing to infections. ${ }^{1,4}$ In contrast, however, diabetes mellitus is a more specific risk factor for mucormycosis. ${ }^{19}$ We thus developed mouse models of intratracheal inoculation with three immunosuppressive conditions that mimic the risk factors (neutropenia, diabetes mellitus, and corticosteroids). After histopathology analysis of the pulmonary lesions, we developed a computer-assisted method to quantify the spores in the pulmonary tissue and to refine the localization of the spores with transmission electron microscopy (TEM).

\section{Materials and Methods}

\section{Reagents}

All of the reagents were purchased from Sigma-Aldrich (Saint-Quentin Fallavier, France) unless otherwise specified.

\section{Mold Strains}

The A. fumigatus reference strain Af293 (CBS 101355; Centraalbureau voor Schimmels Cultures, Utrecht, the Netherlands) was selected. ${ }^{20}$ In the absence of a consensual reference strain for L. corymbifera, a clinical strain (CNRMA 11.1047; Institut Pasteur, Paris, France) isolated from lung lesions of a neutropenic patient with proven invasive mucormycosis was used. Endospores of L. corymbifera and conidia of A. fumigatus (thereafter designated spores) were stored frozen in milk at $-80^{\circ} \mathrm{C}$ and subcultured on potato dextrose agar. Spores were harvested by scrapping 6-day subcultures on potato dextrose agar slants at $30^{\circ} \mathrm{C}$. Spore suspensions in sterile phosphate-buffered saline (PBS) that contained $0.05 \%$ Tween 80 were filtered through a nylon filter (11- $\mu \mathrm{m}$ pore size) to remove hyphae and agglutinated spores and were enumerated with a hemacytometer.
Appropriate dilutions were then made in PBS. Spore suspension was kept at $>4^{\circ} \mathrm{C}$ for $<24$ hours until use.

\section{Animals}

Outbred male OF1 mice ( 8 weeks; Charles River, L'Arbresle, France) were housed seven per cage in our animal facilities and given food and water ad libitum. This study was conducted in accordance with the European Union guidelines for animal care (Directive 2010/63/UE; September 22, 2010), and experimental protocols were approved by the Institut Pasteur Animal Experimentation Ethics Committee (CETEA 2013-0135).

\section{Experimental Infection}

Each immunosuppressive drug was administered via the intraperitoneal route to groups of three mice each. Corticosteroid-induced immunosuppression was achieved by administration of cortisone acetate $(10 \mathrm{mg}$ suspended in PBS that contained 1\% Tween 20) 72 and 24 hours before inoculation. ${ }^{21}$ Administration of cyclophosphamide (100 $\mathrm{mg} / \mathrm{kg}$ in PBS), 96 and 24 hours before inoculation, ${ }^{22}$ produced neutropenia as assessed by leukocyte counts with the use of an automated hematology analyzer (scil vet $\mathrm{ABC}$, Altorf, France) on the day of inoculation. Type 1 diabetes was induced by the administration of streptozocin $(200 \mathrm{mg} / \mathrm{kg}$ in $0.2 \mathrm{~mL}$ of ice-cold citrate buffer, $\mathrm{pH}$ 4.5). ${ }^{23}$ Because OF1 mice reacted differently to streptozocin than $\mathrm{C} 57 \mathrm{BL} / 6$ or $\mathrm{BALB} / \mathrm{c}$ mice, diabetes developed in 2 days instead of 7 days (with death from diabetes occurring between 3 and 10 days after injection of streptozocin). Glycemia was assessed with a glucometer (Accu Check Aviva; Roche Diagnostics, Meylan, France). Only OF1 mice with a glucose concentration $>16.6 \mathrm{mmol} / \mathrm{L}$ at day 2 after streptozocin injection (ie, $85 \%$ of the mice injected with streptozocin) were inoculated.

Intratracheal inoculation of $50 \mu \mathrm{L}$ of PBS with $0.05 \%$ Tween 80 that contained $1 \times 10^{7}$ spores [spore viability (means $\pm \mathrm{SD}$ ), $98.6 \% \pm 2.7 \%]^{24}$ was performed with a blunt-end, 24-gauge needle after isoflurane (Iso-Vet; Piramal Healthcare, London, United Kingdom) anesthesia. The mice were sacrificed by carbon dioxide inhalation at 1 , 6 , and 18 hours after inoculation (hpi), and the lungs were harvested. Because it was impossible to do all of the experimentations at the same time, the same batch of mice was used for a given underlying condition, and all of the experiments were performed over a 3-month period.

\section{Lung Histopathology}

The lungs were fixed in 10\% neutral-buffered formalin and embedded in paraffin. Four-micrometer sections were cut in the middle of the paraffin block and stained with hematoxylin and eosin to describe histologic lesions 


\section{A Tissue inflammation}

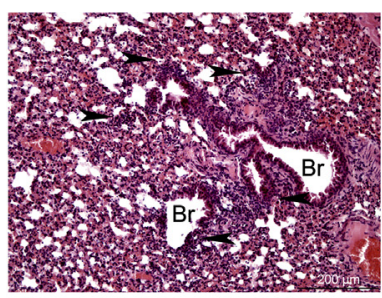

A. fumigatus

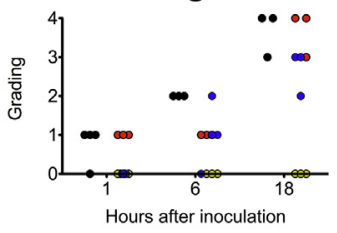

B Spore phagocytosis

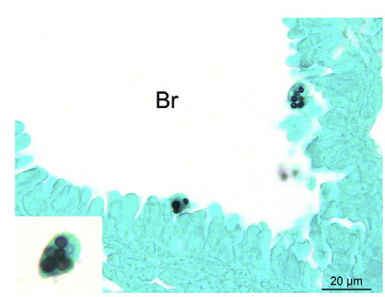

A. fumigatus

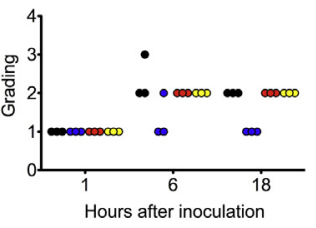

C Spore alteration

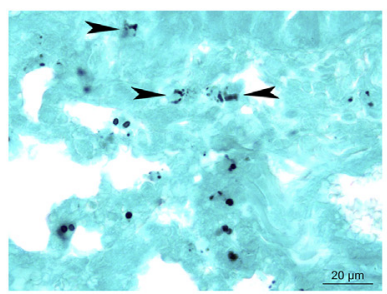

A. fumigatus

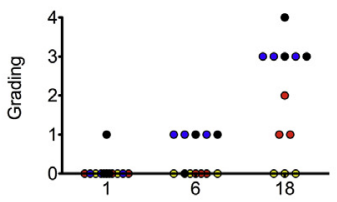

Hours after inoculation

\section{Spore germination}

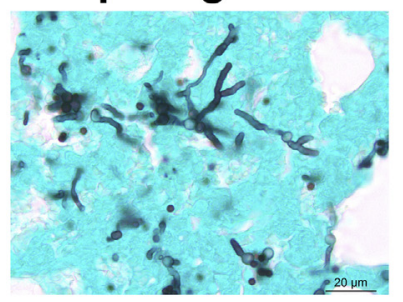

\section{A. fumigatus}

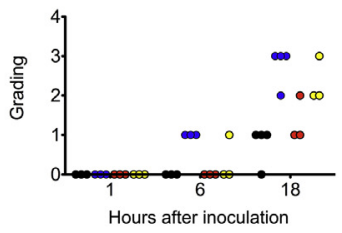

L. corymbifera

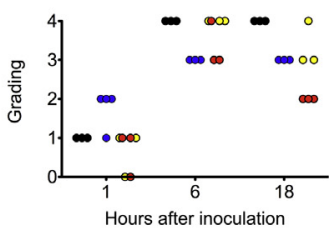

L. corymbifera

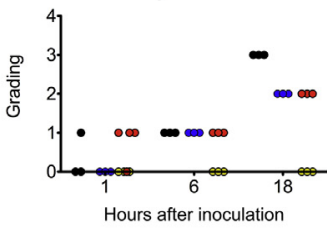

Figure 1 Comparison of early pulmonary events observed after inoculation of Aspergillus fumigatus or Lichtheimia corymbifera to mice with various underlying conditions. Groups of OF1 mice were either untreated or immunosuppressed by cortisone acetate, streptozocin (diabetes), or cyclophosphamide (neutropenia) and were inoculated intratracheally with $1 \times 10^{7} \mathrm{~A}$. fumigatus and $L$. corymbifera. Lungs were harvested at 1,6 , and 18 hours after inoculation and were processed for histopathologic examination. The same experimented operator (G.J.) blindly performed a grading of various variables by using a 5 -grade scale from 0 to 4 (0, none; 1 , minimal; 2, mild; 3, moderate; 4, marked). Each symbol represents a mouse. A: Inflammatory infiltrates (arrowheads) caused by A. fumigatus are characterized by inflammatory cells surrounding bronchi or alveoli. B: Clusters of $L$. corymbifera spores are surrounded by a cell membrane, suggesting indirect evidence of phagocytosis. C: Structural alteration of $L$. corymbifera spores (arrowheads). D: Ultimate stage of $A$. fumigatus germination is represented by fungal hyphae invading the lung parenchyma. $n=3$ OF1 mice per group. Hematoxylin and eosin staining (A); Grocott methenamine silver staining $(\mathbf{B}-\mathbf{D})$. $\mathrm{Br}$, bronchi.

\section{L. corymbifera}

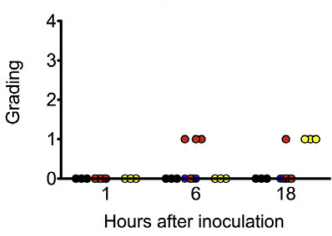

untreated

(distribution of lesions, inflammation, necrosis) and Grocott methenamine silver for the detection of fungi. One slide that included the six lobes was analyzed for each mouse. The analysis was performed by a single member of the group (G.J.) blinded to the fungal species and the underlying condition. Qualitative histologic changes were recorded and semiquantitatively scored with a 5 -grade scale as previously reported $(0$, none; 1 , minimal; 2, mild; 3, moderate; 4, marked). ${ }^{25,26}$ The scoring took into account tissue damage (necrosis and inflammation characterized by infiltration of neutrophils, lymphocytes, and macrophages), fungal changes on the basis of structural characteristics (altered fungal wall, spore germination, presence of hyphae), and indirect evidence for phagocytosis (spore clustering surrounded by a cell membrane). Silver-stained slides were then digitized
(Axioscan Z1; Zeiss, Marly Le Roi, France) for computerassisted morphometric analysis.

\section{Computer-Assisted Morphometric Analysis Using Icy}

Icy is an open community platform for bioimage informatics. ${ }^{27}$ A specific plugin called mycosis lung quantifier was developed to quantify and analyze the lung distribution of fungal spores. Plugin and its source code are available (http://icy. bioimageanalysis.org/plugin/Mycosis_Lung_Quantifier, last accessed April 8, 2015). Because the differentiation between bronchi and bronchioles were difficult on silver-stained slides, we delineated manually all of the sections surrounded by a cubic epithelium, thereafter designated bronchus. The plugin allocated each spore to the closest bronchus section (Supplemental Figure S1). Red lines tracked the spores inside 


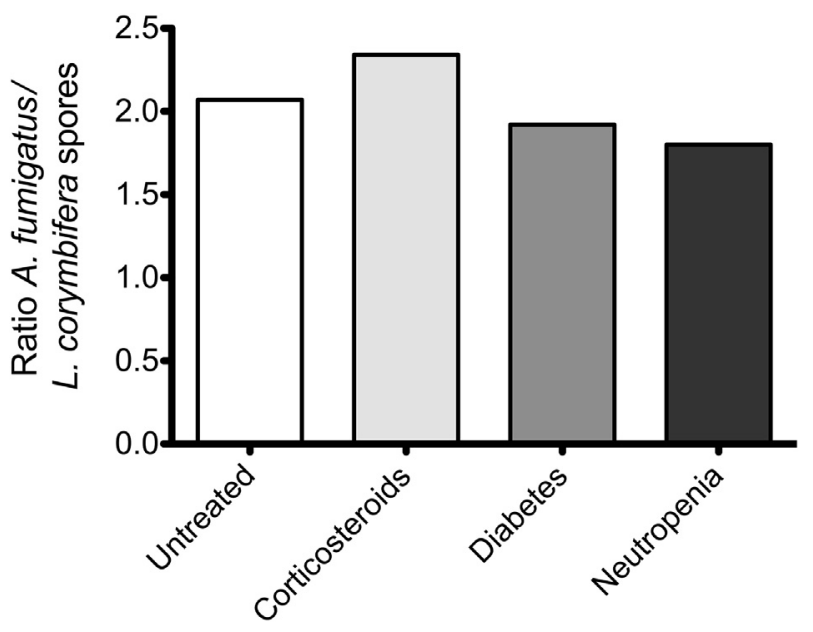

Figure 2 Aspergillus fumigatus spore count is twofold higher in the alveoli around the bronchi than Lichtheimia corymbifera at 1 hour after inoculation of OF1 mice. Four groups of OF1 mice were intratracheally inoculated with $1 \times 10^{7} \mathrm{~A}$. fumigatus or L. corymbifera, and euthanized 1 hour after inoculation for lung histopathologic analysis. One lung section per mouse was analyzed. The mean number of spores per $10^{5}$ bronchial surface units in pixels (mean spores/BSU) was counted by using the mycosis lung quantifier Icy plugin in the alveoli (ie, at a distance $>16$ and $<320$ pixels from the apical pole of the bronchi). Each bar represents the fold increase of the mean alveolar spores/bronchial surface units of $A$. fumigatus compared with that of L. corymbifera. No difference was found between the underlying conditions $(P=0.39$, analysis of variance test). $n=3$ OF1 mice per group.

the bronchus lumen by measuring a negative distance from the apical pole of the bronchial epithelium delineation. Yellow lines measured a positive distance between spores and the apical pole of the bronchial epithelium. Distances are expressed in pixels (px) and are not converted to micrometers for accuracy.

\section{Image Processing}

The same person (B.R.) blindly processed all of the slides. The first step was to digitize the lung slides (Axioscan Z1; Zeiss) by using $\times 20$ magnification. The image size was reduced to $40 \%$ of the original size by using Zen lite 2012 software version 2.1 64-bit (Zeiss), and the image file was converted into the tiff format. The maximum image size that can be processed by Icy depends on the computer configuration. The minimal required configuration for the computer by using Icy plugin was $16 \mathrm{Mb}$ random access memory and 256 Go of memory to allow processing of images $<1$ Go. After loading the image on Icy, a preliminary step consisted of bronchial lumen surface delineation and artifact exclusion. With the use of the region of interest area tool on Icy, delineation of each bronchus surface was performed manually on the whole surface of the lung section. Bronchus section was considered as such when cubic epithelium was present, even if the section was not perfectly round. Artifacts such as silver staining of the vascular connective tissue were excluded with the black paintbrush.
Plugin Variables Choice

The chosen variables are described below.

\section{Threshold}

The sensibility of spore detection depends on the silver staining of each lung section. In fact, if the staining is highly contrasted, the detection threshold has to be lowered to detect only spores. A perfect methenamine silver staining is needed to avoid black artifacts that hamper Icy counting. For each slide, different thresholds were tested, and the optimal threshold was visually determined, ranging from 20 to 110 , according to the silver uptake of each slide.

\section{Size}

Minimal and maximal sizes were chosen to optimize the detection of each spore at different germination stage. Minimal and maximal sizes were 0 to $20 \mathrm{px}$ and $1000 \mathrm{px}$, respectively.

\section{Epithelium Width}

To enumerate the spores inside the bronchial epithelium, we had to determine the epithelium width. The size of the bronchial epithelium was measured on 10 randomly chosen bronchi from 10 different images, using the ruler helper plugin in Icy (http://icy.bioimageanalysis.org/plugin/Ruler Helper, last accessed March 25, 2014). The mean epithelium size was $32 \mathrm{px}$ (range, 16 to $49 \mathrm{px}$ ). The smallest epithelium width, $16 \mathrm{px}$, was chosen to avoid unduly counting some spores as intraepithelial.

\section{Maximal Distance}

Our purpose was not to quantify all of the spores present in the lungs but to localize spores inside and around bronchi. Furthermore, Grocott methenamine silver stained in black the edges of the lungs, leading to artifacts. The maximal distance from the bronchial lumen was then reduced. We chose $320 \mathrm{px}$ from the apical pole of the attributed bronchus, 10-fold the mean epithelium width, to give a large overview of the distribution of the fungi around bronchi. The maximal number of spores was detected before the limit of 320 px on 12 randomly chosen slides (Supplemental Figure S2A).

\section{Bronchial Surface and Perimeter}

Anatomical or experimental variations during lung processing can occur. The number of bronchus sections by slide varies according to the position of the lungs when paraffinembedded. The mean number of bronchus sections was 66 (range, 22 to 123) in all of the lung slides, and the bronchus surface varied according to their level in the respiratory tract but also according to the cutting section. To standardize bronchi, we decided to exclude smallest and largest bronchi, based on both perimeter and surface measure. The smallest bronchi with a perimeter $<300 \mathrm{px}$ and/or a surface $<5000 \mathrm{px}$ can widely be open on the alveoli, leading to false-positive detection of bronchial spores, and the bronchial epithelium 


\section{Control}
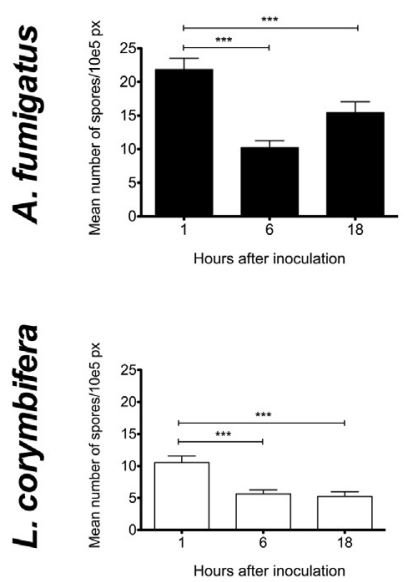

Corticosteroids
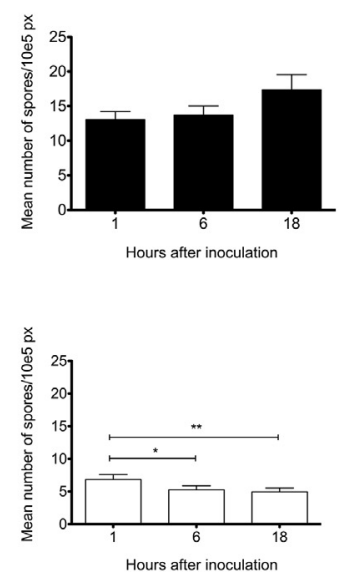

Diabetes
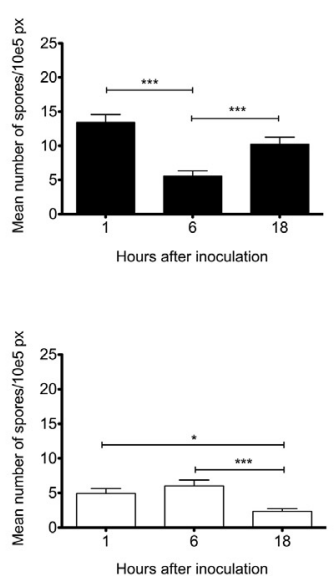

Neutropenia
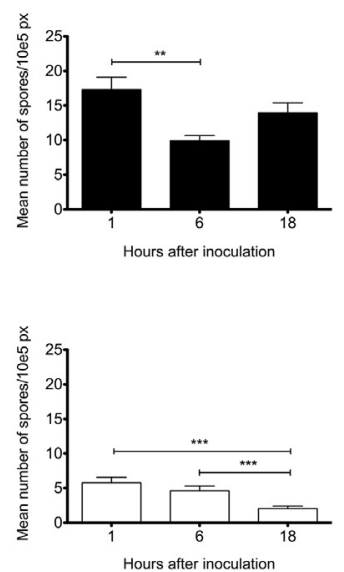

Figure 3 Distribution of Aspergillus fumigatus and Lichtheimia corymbifera spores inside bronchi over time. Four groups of OF1 mice were intratracheally inoculated with $1 \times 10^{7} \mathrm{~A}$. fumigatus or L. corymbifera and were euthanized at 1,6 , or 18 hours after inoculation for lung histopathologic analysis. One lung section per mouse was analyzed. A morphometric analysis was performed with mycosis lung quantifier Icy plugin. Bars represent the mean number of bronchial spores per $10^{5}$ bronchial surface units in pixels. $n=3$ OF1 mice per group. ${ }^{*} P<0.05,{ }^{*} P<0.01$, and ${ }^{* * *} P<0.001$ (Mann-Whitney test) versus bronchial spores from the other fungus compared at each time point. px, pixel.

was too thin to allow reliable counting. The largest bronchi with a perimeter $>10,000 \mathrm{px}$ and a surface $>200,000 \mathrm{px}$ were excluded because of an excessive silver uptake by mucusproducing cells, leading to black artifacts. Out-of-range bronchi were colored in red, and spores attributed to these bronchi were also excluded.

The data generated by the plugin were automatically exported as Excel files (Excel version 14.5.2; Microsoft, Redman, WA), reporting for each bronchus analyzed the number of spores, the spore distance in pixels from the bronchi, and chosen variables and the reasons of bronchi exclusion.

\section{Plugin Variables Validation}

Each plugin variable was validated by using randomized blinded lung sections (B.R.). We first determined whether Icy counting was efficient to identify spores inside bronchi, either in the bronchial lumen or in the bronchial epithelium. We validated the method that enumerated visually all spores present in the lumen or in the epithelium of 60 bronchi (10 bronchi/ mouse). Icy count was not significantly different from the visual count (Supplemental Figure S2B). We then checked if the contrast of the lung section could influence the detection of spores by Icy. The detection threshold was visually subjectively chosen. We compared three different thresholds (from 30, highly contrasted slides, to 90, low-contrasted slides) from six different slides (two per threshold) by using visual and Icy counting. The detection threshold did not significantly influence Icy counting (Supplemental Figure S2C). With the use of these same sections, we raised the question of the influence of threshold variations on spore count (Supplemental Figure S2D). Slight variations of the threshold $( \pm 20)$ did not significantly change the spore count.

Two categories of spores were defined according to their location from the apical pole of the bronchial epithelium as follows: The bronchial spores, including the luminal spores (harboring a negative distance value and therefore clearly not internalized by the respiratory epithelium), and the epithelial spores located between 0 and $16 \mathrm{px}$, corresponding to either spores at the apical pole of the epithelium or spores that could be internalized by the epithelium; and the alveolar spores, located between $>16 \mathrm{px}$ and $<320 \mathrm{px}$ from the apical epithelium pole of the attributed bronchus, knowing that some spores were in the interstitial spaces.

\section{TEM}

For ultrastructural studies, corticosteroid treatment and a higher inoculum size $\left(5 \times 10^{7}\right.$ spores $)$ were used. The right lung was cut into $2-\mathrm{mm}$ pieces, fixed for 48 hours at $4{ }^{\circ} \mathrm{C}$ in $0.2 \mathrm{~mol} / \mathrm{L}$ sodium cacodylate buffer, $\mathrm{pH} 7.4$, that contained $2.5 \%$ glutaraldehyde and $0.5 \%$ paraformaldehyde. Specimens were then washed three times in sodium cacodylate buffer $(0.2 \mathrm{~mol} / \mathrm{L}, \mathrm{pH} 7.4)$, after being fixed 1 hour with $1 \%$ osmium tetroxide in $0.2 \mathrm{~mol} / \mathrm{L}$ sodium cacodylate buffer ( $\mathrm{pH}$ 7.4) and washed three times (10 minutes) in sodium cacodylate buffer $(0.2 \mathrm{~mol} / \mathrm{L}, \mathrm{pH} 7.4)$. Dehydration (graded ethanol and acetone) and resin infiltration (acetone and epon resin mixture) were performed with automated microwaves (Leica). Specimens were embedded in epoxy resin for 2 hours in automated microwaves. Thin sections of $70 \mathrm{~nm}$ were cut with a UC6 ultramicrotome (Leica, Wetzlar, Germany) and stained with uranyl acetate and lead citrate. Specimen sections were observed at $80 \mathrm{kV}$ with a Hitachi H-7650 TEM (Hitachi, Yokohama, Japan). A total of four grids with 200 to 500 spores/grid were observed per mold.

\section{Statistical Analysis}

The objective of the computer-assisted morphometry was to analyze spore distribution at the bronchial level. We used 


\section{A. fumigatus}
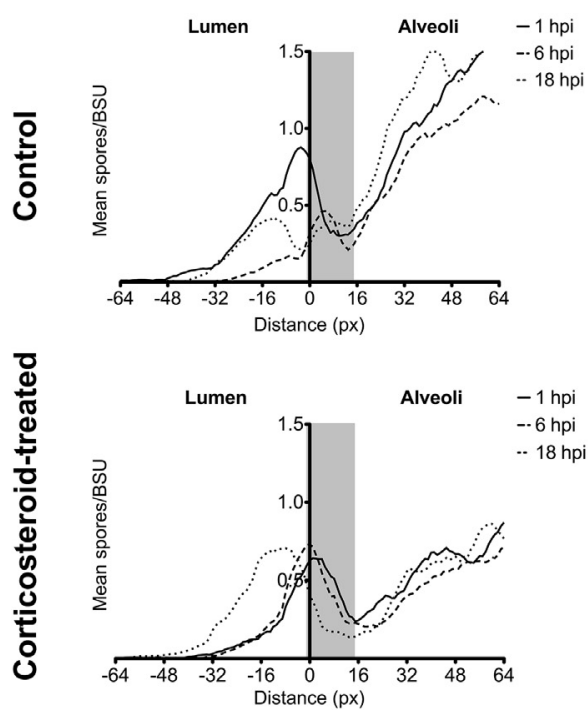

\section{L. corymbifera}
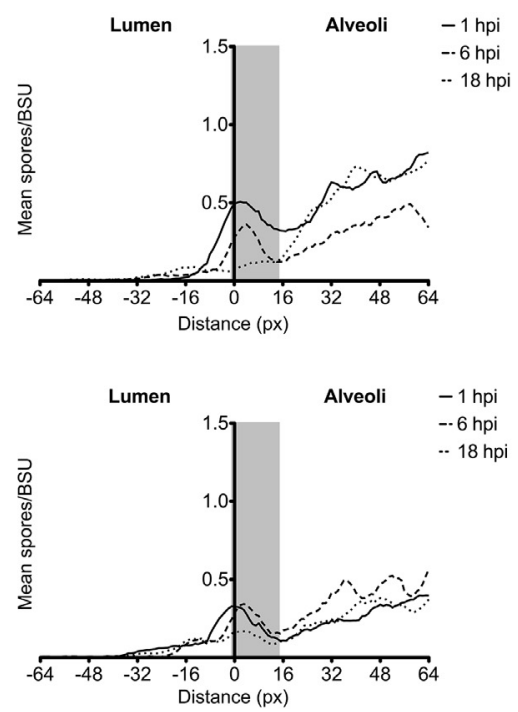

Figure 4 Distribution of spores in the lungs relative to the bronchial epithelium in mice infected by Aspergillus fumigatus or Lichtheimia corymbifera. Groups of untreated and corticosteroid-treated mice were euthanized, and lungs were collected at 1,6 , and 18 hpi of $10^{7}$ spores of $A$. fumigatus or $L$. corymbifera. Spores located at a maximal distance of $320 \mathrm{px}$ from the apical pole of the bronchial epithelium were enumerated on silver-stained sections by using the mycosis lung quantifier Icy plugin. The mean of spores per $10^{5} \mathrm{BSU}$ (in px) located between $-64 \mathrm{px}$ and $64 \mathrm{px}$ is represented as a curve. The gray area represents the epithelium width. The mean spores/BSU curve at $1 \mathrm{hpi}$ is superimposed or above the other curves beyond the limit of $16 \mathrm{px}$, suggesting that spores do not accumulate over time at the basal pole of the epithelium. $n=3$ mice per group. BSU, bronchial surface unit; hpi, hours after inoculation; px, pixel. the total bronchial surface of each lung section to perform our analysis. Indeed, each mouse had $65.4 \pm 16.2$ bronchial sections. Because their shape varied according to the orientation of the lungs when paraffin-embedded, we pooled the bronchial surfaces provided by Icy for each group of three mice after checking that spores counts were similar among mice of each group. We then expressed the Icy counts as a mean number of spores per $10^{5}$ bronchial surface units (BSUs) in pixels (mean spores/BSU) \pm SEM. To compare bronchial surfaces of each group, we used the oneway analysis of variance parametric test after checking the equality of variances. Bronchial distributions of spores according to molds and conditions on Icy analysis were compared with nonparametric tests (Kruskal-Wallis or Mann-Whitney test). For multiple comparisons, Bonferroni correction was applied. For the analysis of the epithelium barrier crossing, the mean spores/BSU located between -64 px and 64 px was analyzed for each condition tested. Curves were smoothed by using eight neighbors' points. All of the statistical analyses were performed with GraphPad Prism version 5.0 (GraphPad Inc., San Diego, CA).

\section{Results}

\section{Underlying Conditions Differently Affect Tissue and} Fungal Damages in Mice Inoculated with $A$. fumigatus Compared with $L$. corymbifera

In preliminary experiments, we assessed the reproducibility of the results at $6 \mathrm{hpi}$ by repeating the experiments in groups of three mice. Subsequent experiments were thus performed once for ethical reasons. For a given condition, time, and mold, the three mice tested per group displayed tissue damages and fungal changes with $<1$ grade in difference on the 5-grade scale used. We therefore considered as relevant any difference of $>2$ grades when comparing the different times and conditions. Relevant variations were recorded over time per fungi for a given underlying condition, especially comparing 1 hpi with 18 hpi. Few events occurred before 6 hpi. Because the viability of inoculums was close to $100 \%$, the differences observed between fungi and conditions cannot be due to variations of the inoculum.

Albeit moderate, inflammation caused by A. fumigatus was higher than that caused by L. corymbifera (Figure 1A). When present, inflammatory cells mainly gathered at the bronchus periphery for A. fumigatus, whereas for L. corymbifera inflammatory cells were sparsely distributed all over the lung parenchyma. Of note, necrosis was present in neutropenic mice instead of inflammation for both molds. Indirect evidence for phagocytosis was significantly more often observed for L. corymbifera than for A. fumigatus whatever the underlying condition (Figure 1B). Alteration of fungal structures was seen, starting at $6 \mathrm{hpi}$ in untreated and corticosteroid-treated mice and to a lesser extent at $18 \mathrm{hpi}$ in the other underlying conditions (Figure 1C). Fungal alteration was similar for both molds, except in neutropenic mice whereby $L$. corymbifera was more altered at $18 \mathrm{hpi}$ than A. fumigatus. Germination of L. corymbifera spores was only observed in diabetic and neutropenic mice, whereas germination of A. fumigatus spores was observed in all underlying conditions (Figure 1D).

Thus, tissue damages and spore germination increased over time for both fungi. A. fumigatus gave more inflammation, germinated more, and was less phagocytized than L. corymbifera. In untreated mice, both molds generated tissue damages, indirect evidence of phagocytosis, and higher fungal alteration score. Diabetes provided a similar profile of tissue damage and spore germination with both molds. The corticosteroid model led to more differences between the two fungi than the other underlying conditions. Neutropenia was the condition associated with the best germination and was hardly associated with fungal alteration for both molds. 

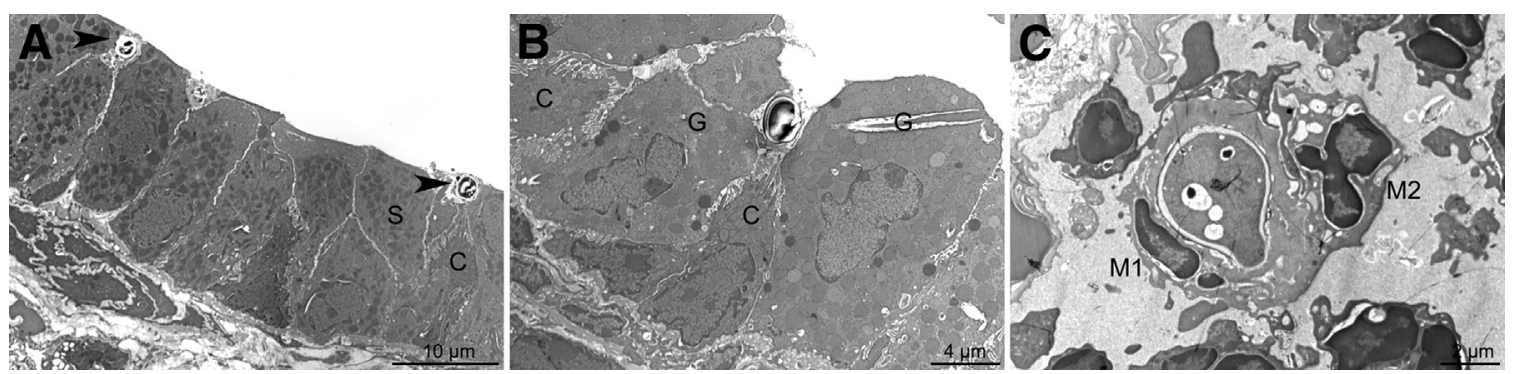

Figure 5 Interaction between Aspergillus fumigatus or Lichtheimia corymbifera spores and bronchial cells in TEM at 6 and 18 hours after inoculation of corticosteroids-treated OF1 mice. OF1 mice were immunosuppressed by cortisone acetate. Lungs were harvested at 6 and 18 hours after intratracheal inoculation of $5 \times 10^{7}$ spores/mouse of $A$. fumigatus or L. corymbifera. Lungs were then processed for TEM. A. fumigatus spores (A, arrowheads) and L. corymbifera spores (B) were not internalized by bronchial epithelial cells but were localized at the junction between serous, ciliated, and/or goblet cells. C: Two macrophages (M1 and M2) in an alveolar space. One spore that has started to germinate is seen phagocytized by the M1 macrophage. C, ciliated cell; G, goblet cell; $\mathrm{S}$, serous cell; TEM, transmission electronic microscopy.

\section{Quantification of Bronchial Spores Using a Computer-Assisted Morphometric Analysis}

We first assessed that the total bronchial surfaces per group of three mice were not different between the four underlying conditions for A. fumigatus and L. corymbifera $(P=0.09$ and $P=0.12$, respectively). We then looked at the bronchial, epithelial, and alveolar distribution of spores within the limit of $320 \mathrm{px}$ around the bronchi. The proportion of alveolar spores varied from $80 \%$ to $95 \%$ according to the underlying condition for both fungi (Supplemental Figure S3). Despite the same inoculum, at 1 hpi the mean alveolar spores/BSU of $A$. fumigatus was twofold that of $L$. corymbifera whatever the underlying condition (Figure 2). The mean bronchial spores/BSU of A. fumigatus was also higher than that of L. corymbifera (Figure 3). The mean bronchial spores/ BSU decreased over time for L. corymbifera for all of the underlying conditions (Figure 3). Although the general trend for A. fumigatus bronchial spores was also a decrease when comparing 1 hpi with 18 hpi for the different underlying conditions (except in corticosteroidtreated mice), an increase was always seen between 6 and 18 hpi. The mean epithelial spores/BSU decreased between 1 and 18 hpi with a 1 hpi:18 hpi ratio of 3.1 in corticosteroid-treated $(P<0.0001), 1.6$ in diabetic $(P=0.002)$, and 2.9 in neutropenic $(P<0.0001)$ mice for A. fumigatus, and 2.9 in control $(P<0.0001), 1.6$ in corticosteroid-treated $(P=0.0008), 2.7$ in diabetic $(P=0.0008)$, and 11.2 in neutropenic $(P<0.0001)$ mice for L. corymbifera.

Because the mean epithelial spores/BSU decreased over time for both fungi, one hypothesis of spore elimination from the bronchi could be the crossing of the epithelium to reach the alveoli/interstitium. Because the epithelium width was considered to be $16 \mathrm{px}$, any crossing of the epithelium should be evidenced by an accumulation of spores over time beyond the limit of $16 \mathrm{px}$. We did not find such accumulation of spores irrespective of the fungus and the underlying condition considered (Figure 4).

\section{Fungi Are Not Internalized by Bronchial Epithelial Cells}

We performed a TEM analysis to find the precise localization of epithelial spores. We chose the corticosteroid condition because corticosteroid-treated mice showed the highest number of spores associated with the bronchial epithelium. We observed the presence of unbound spores in the lumen, mainly at $6 \mathrm{hpi}$; the presence of spores in close contact with the bronchial epithelial cells; the presence of numerous spores inside mononuclear phagocytic cells; and the absence of internalization by epithelial cells at 6 and $18 \mathrm{hpi}$. When the spores were in contact with epithelial cells, they were mainly located at the junction between ciliated and/or goblet cells (Figure 5, A and B). Inside mononuclear phagocytic cells, spores of A. fumigatus were seen with germ tubes inside the cytoplasm (Figure 5C), whereas no germination of $L$. corymbifera spores was observed, although they were swollen from 2 to $4 \mu \mathrm{m}$. Of note, no spore was observed inside type 2 pneumocytes whatever the time.

\section{Discussion}

Few in vivo studies have simultaneously compared A. fumigatus with Mucorales either for pathophysiologic ${ }^{28,29}$ or pharmacologic purposes. ${ }^{30}$ Because our main interest was the early interactions between spores and bronchial/bronchiolar epithelium to explain the broncho-invasive forms of invasive mold infections, we decided to study these interactions in animal models with the use of classicl histopathology and ultrastructural microscopy at selected time points that compared both fungi. To avoid a major limitation in classic histopathology analysis, which is the lack of objective quantification of the observations, we used Icy, an open community platform for bioimage informatics, and designed a specific plugin to quantify and localize fungal spores relative to bronchial structures in the lung of infected mice. This allowed us to evaluate reproducibility and to compare multiple variables, including four underlying conditions, two molds, and three times after inoculation.

We observed more A. fumigatus spores around the alveoli and inside the bronchi than $L$. corymbifera spores. 
As a corollary, $L$. corymbifera spores were more sparsely distributed all over the lung parenchyma than A. fumigatus spores. Although these spores have the same size, we can hypothesize that A. fumigatus and L. corymbifera probably have different surface adhesins that confer variable binding properties to bronchial epithelial cells. Few data exist on Lichtheimia spp. cell wall composition, ${ }^{31}$ whereas another member of the Mucorales, Rhizopus arrhizus (oryzae), is studied more. Chitin and other polysaccharides such as glucans and mannans are major components of the fungal cell wall; their composition varies according to the stage of growth. ${ }^{32}$ Chitin and glucan synthase genes are also highly different between R. arrhizus and A. fumigatus. ${ }^{33}$ To date, binding factors such as galactosaminogalactan have only been identified in A. fumigatus, ${ }^{34}$ whereas both $R$. arrhizus and A. fumigatus have the capacity to bind to laminin and type IV collagen. ${ }^{35,36}$ A comparative analysis of A. fumigatus and $L$. corymbifera cell wall could help our understanding of bronchial epithelial cell adhesion. By analogy with the receptor to Mucorales found on the endothelial cells in diabetic conditions, ${ }^{37}$ host factors that have to be determined could also influence the persistence of $A$. fumigatus rather than L. corymbifera in the bronchi.

Thanks to the mycosis lung quantifier plugin we also observed the increase of A. fumigatus spores in the bronchi at 18 hpi in all of the underlying conditions, whereas L. corymbifera spore number decreased over time. Because A. fumigatus spores were mainly distributed around the bronchi, we can hypothesize that their elimination through the bronchial tract was enhanced, possibly through mechanical factors such as cough and the mucociliary escalator. Distributed deeper in the lungs, $L$. corymbifera may have been altered on site and eliminated to a lesser extent via the bronchi. Different mechanisms may explain the absence of bronchial spore decrease at $6 \mathrm{hpi}$ in the corticosteroid-treated mice infected with A. fumigatus. Mucus composition of bronchial epithelium could have been altered by corticosteroids by impairing the elimination of A. fumigatus spores, ${ }^{38}$ or, as spores were less phagocytized, they may present an earlier stage of germination (ie, earlier changes in their cell wall composition) to unmask different pathogen-associated molecular patterns, ${ }^{32}$ leading to epithelial cell adhesion. ${ }^{39}$ Our models allowed us to observe with TEM at least 200 spores in the lung parenchyma. Despite these high numbers, we did not notice in vivo internalization of spores by respiratory epithelial cells in the first 18 hours after intratracheal challenge, neither for A. fumigatus nor for L. corymbifera. This does not definitively rule out this potential initial event in the mold infection because TEM is notoriously poorly sensitive. However, given the high number of spores observed inside the bronchi, we can conclude that if early internalization occurs, this is a rare phenomenon. We cannot exclude either that our experimental conditions, in particular the high spore concentrations used to optimize analysis of structural characteristics, ${ }^{40}$ may have triggered reactions that prevented spore internalization. This lack of internalization contrasts with the easy/frequent internalization of fungal spores by cell lines in vitro, either primary epithelial, ${ }^{11,41}$ bronchial, or alveolar epithelial cell lines. ${ }^{42}$ The discrepancy between in vitro and in vivo observations may be due to the complex microenvironment present in vivo but not in vitro. Thus, long pentraxin 3 or mannan-binding lectines may help prevent internalization by epithelial cells while enhancing macrophage phagocytosis. ${ }^{43,44}$ Because addition of Tween 80 is necessary to prevent aggregation of fungal spores during both in vitro and in vivo experiments even for aerosolized spores, ${ }^{45}$ it cannot explain the absence of in vivo internalization but can change the interactions between the spores and the host cells. However, it did not prevent observing differences between both fungi.

Our results are consistent with previous studies about the role of professional phagocytic cells in antifungal defense. $^{28,46}$ Spores were phagocytized in the bronchi by mononuclear phagocytic cells that are difficult to accurately identify with TEM. ${ }^{47}$ Alveolar macrophages are recognized as the main phagocytic cells involved in spore engulfment. ${ }^{28,46}$ They may colonize bronchi, too, but their number in the normal bronchi is difficult to evaluate. ${ }^{48}$ Alveolar macrophages are long-lived cells relatively not affected by radiotherapy or chemotherapy, ${ }^{49}$ but their phagocytic functions can be altered by corticosteroid use and diabetes. ${ }^{50,51}$ Here, we showed that the two underlying conditions in which $L$. corymbifera spores can germinate were diabetes and neutropenia. We already know that alveolar macrophages from diabetic mice are unable to inhibit mucorale germination in vitro. ${ }^{28}$ Because $L$. corymbifera spore phagocytosis in our diabetic mice was comparable with other underlying conditions, we can hypothesize that a killing defect and not altered phagocytosis could explain the development of mucormycosis in diabetic patients.

\section{Conclusion}

The systematic comparison of various timings and underlying conditions confirms differences between the two molds in terms of kinetics and extent of tissue lesions induced, phagocytosis, and germination even at the early stages of lung infection. Bioimage informatics provides objective data on the bronchi interaction with fungal spores. A. fumigatus seems to better adapt to its host than L. corymbifera, partly explaining the higher frequency of aspergillosis in the hematologic setting. Further studies that compare both molds are needed to explore fungal components involved in bronchial adhesion differences between A. fumigatus and L. corymbifera.

\section{Acknowledgments}

We thank Huot Khun for technical assistance with histology.

B.R., G.J., F.C., F.D., and S.B. conceived the experiments; B.R., F.d.C., and J.-C.O.-M., conceived the plugin; B.R., G.J., D.G.-H., and C.S. performed experiments; B.R., G.J., C.R., F.D., and S.B. analyzed data. All authors wrote 
and approved the submitted and published versions of the manuscript.

\section{Supplemental Data}

Supplemental material for this article can be found at http://dx.doi.org/10.1016/j.ajpath.2015.04.027.

\section{References}

1. Lortholary O, Gangneux JP, Sitbon K, Lebeau B, de Monbrison F, Le Strat Y, Coignard B, Dromer F, Bretagne S: Epidemiological trends in invasive aspergillosis in France: the SAIF network (2005-2007). Clin Microbiol Infect 2011, 17:1882-1889

2. Bitar D, Van Cauteren D, Lanternier F, Dannaoui E, Che D, Dromer F, Desenclos JC, Lortholary O: Increasing incidence of zygomycosis (mucormycosis), France, 1997-2006. Emerg Infect Dis 2009, 15:1395-1401

3. Kontoyiannis DP, Marr KA, Park BJ, Alexander BD, Anaissie EJ, Walsh TJ, Ito J, Andes DR, Baddley JW, Brown JM, Brumble LM, Freifeld AG, Hadley S, Herwaldt LA, Kauffman CA, Knapp K, Lyon GM, Morrison VA, Papanicolaou G, Patterson TF, Perl TM, Schuster MG, Walker R, Wannemuehler KA, Wingard JR, Chiller TM, Pappas PG: Prospective surveillance for invasive fungal infections in hematopoietic stem cell transplant recipients, 20012006: overview of the Transplant-Associated Infection Surveillance Network (TRANSNET) Database. Clin Infect Dis 2010, 50: $1091-1100$

4. Lanternier F, Dannaoui E, Morizot G, Elie C, Garcia-Hermoso D, Bitar D, Huerre M, Dromer F, Lortholary O; French Mycosis Study Group: A global analysis of mucormycosis in France: the RetroZygo study (2005-2007). Clin Infect Dis 2012, 54(Suppl 1):S35-S43

5. Petrikkos G, Skiada A, Lortholary O, Roilides E, Walsh TJ, Kontoyiannis DP: Epidemiology and clinical manifestations of mucormycosis. Clin Infect Dis 2012, 54(Suppl 1):S23-S34

6. Bergeron A, Porcher R, Sulahian A, de Bazelaire C, Chagnon K, Raffoux E, Vekhoff A, Cornet M, Isnard F, Brethon B, Lacroix C, Poirot JL, Bouges C, Derouin F, Tazi A, Ribaud P: The strategy for the diagnosis of invasive pulmonary aspergillosis should depend on both the underlying condition and the leucocyte count of patients with hematological malignancies. Blood 2012, 119:1831-1837

7. Logan PM, Primack SL, Miller RR, Müller NL: Invasive aspergillosis of the airways: radiographic, CT, and pathologic findings. Radiology 1994, 193:383-388

8. Kojima R, Tateishi U, Kami M, Murashige N, Nannya Y, Kusumi E, Sakai M, Tanaka Y, Kanda Y, Mori SI, Chiba S, Kusumoto M, Miyakoshi S, Hirai H, Taniguchi S, Sakamaki H, Takaue Y: Chest computed tomography of late invasive aspergillosis after allogeneic hematopoietic stem cell transplantation. Biol Blood Marrow Transplant 2005, 11:506-511

9. Chamilos G, Marom EM, Lewis RE, Lionakis MS, Kontoyiannis DP: Predictors of pulmonary zygomycosis versus invasive pulmonary aspergillosis in patients with cancer. Clin Infect Dis 2005, 41:60-66

10. Lee FY, Mossad SB, Adal KA: Pulmonary mucormycosis: the last 30 years. Arch Intern Med 1999, 159:1301-1309

11. Khoufache K, Cabaret O, Farrugia C, Rivollet D, Alliot A, Allaire E, Cordonnier C, Bretagne S, Botterel F: Primary in vitro culture of porcine tracheal epithelial cells in an air-liquid interface as a model to study airway epithelium and Aspergillus fumigatus interactions. Med Mycol 2010, 48:1049-1055

12. Paris S, Boisvieux-Ulrich E, Crestani B, Houcine O, Taramelli D, Lombardi L, Latgé JP: Internalization of Aspergillus fumigatus conidia by epithelial and endothelial cells. Infect Immun 1997, 65: $1510-1514$
13. Amitani R, Kawanami R: Interaction of Aspergillus with human respiratory mucosa: a study with organ culture model. Med Mycol 2009, 47(Suppl 1):S127-S131

14. Fekkar A, Balloy V, Pionneau C, Marinach-Patrice C, Chignard M, Mazier D: Secretome of human bronchial epithelial cells in response to the fungal pathogen Aspergillus fumigatus analyzed by differential in-gel electrophoresis. J Infect Dis 2012, 205:1163-1172

15. Tomee JF, Wierenga AT, Hiemstra PS, Kauffman HK: Proteases from Aspergillus fumigatus induce release of proinflammatory cytokines and cell detachment in airway epithelial cell lines. J Infect Dis 1997, 176:300-303

16. Bozza S, Gaziano R, Spreca A, Bacci A, Montagnoli C, di Francesco P, Romani L: Dendritic cells transport conidia and hyphae of Aspergillus fumigatus from the airways to the draining lymph nodes and initiate disparate Th responses to the fungus. J Immunol 2002, 168:1362-1371

17. Amitani R, Murayama T, Nawada R, Lee WJ, Niimi A, Suzuki K, Tanaka E, Kuze F: Aspergillus culture filtrates and sputum sols from patients with pulmonary aspergillosis cause damage to human respiratory ciliated epithelium in vitro. Eur Respir J 1995, 8:1681-1687

18. Filler SG, Sheppard DC: Fungal invasion of normally non-phagocytic host cells. PLoS Pathog 2006, 2:e129

19. Rammaert B, Lanternier F, Poirée S, Kania R, Lortholary O: Diabetes and mucormycosis: a complex interplay. Diabetes Metab 2012, 38:193-204

20. Pain A, Woodward J, Quail MA, Anderson MJ, Clark R, Collins M, Fosker N, Fraser A, Harris D, Larke N, Murphy L, Humphray S, O'Neil S, Pertea M, Price C, Rabbinowitsch E, Rajandream MA, Salzberg S, Saunders D, Seeger K, Sharp S, Warren T, Denning DW, Barrell B, Hall N: Insight into the genome of Aspergillus fumigatus: analysis of a $922 \mathrm{~kb}$ region encompassing the nitrate assimilation gene cluster. Fungal Genet Biol 2004, 41:443-453

21. Sugar AM, Liu XP: Combination antifungal therapy in treatment of murine pulmonary mucormycosis: roles of quinolones and azoles. Antimicrob Agents Chemother 2000, 44:2004-2006

22. Li X, Gao M, Han X, Tao S, Zheng D, Cheng Y, Yu R, Han G, Schmidt M, Han L: Disruption of the phospholipase D gene attenuates virulence of Aspergillus fumigatus. Infect Immun 2012, 80: 429-440

23. Ibrahim AS, Avanessian V, Spellberg B, Edwards JE: Liposomal amphotericin B, and not amphotericin B deoxycholate, improves survival of diabetic mice infected with Rhizopus oryzae. Antimicrob Agents Chemother 2003, 47:3343-3344

24. Hohl TM, Van Epps HL, Rivera A, Morgan LA, Chen PL, Feldmesser M, Pamer EG: Aspergillus fumigatus triggers inflammatory responses by stage-specific beta-glucan display. PLoS Pathog 2005, 1:e30

25. Leymarie O, Jouvion G, Hervé PL, Chevalier C, Lorin V, Lecardonnel J, Da Costa B, Delmas B, Escriou N, Le Goffic R: Kinetic characterization of PB1-F2-mediated immunopathology during highly pathogenic avian H5N1 influenza virus infection. PLoS One 2013, 8:e57894

26. Ibrahim-Granet $\mathrm{O}$, Jouvion $\mathrm{G}$, Hohl $\mathrm{TM}$, Droin-Bergère $\mathrm{S}$, Philippart F, Kim OY, Adib-Conquy M, Schwendener R, Cavaillon JM, Brock M: In vivo bioluminescence imaging and histopathopathologic analysis reveal distinct roles for resident and recruited immune effector cells in defense against invasive aspergillosis. BMC Microbiol 2010, 10:105

27. de Chaumont F, Dallongeville S, Chenouard N, Hervé N, Pop S, Provoost T, Meas-Yedid V, Pankajakshan P, Lecomte T, Le Montagner Y, Lagache T, Dufour A, Olivo-Marin JC: Icy: an open bioimage informatics platform for extended reproducible research. Nat Methods 2012, 9:690-696

28. Waldorf AR, Levitz SM, Diamond RD: In vivo bronchoalveolar macrophage defense against Rhizopus oryzae and Aspergillus fumigatus. J Infect Dis 1984, 150:752-760

29. Waldorf AR, Diamond RD: Neutrophil chemotactic responses induced by fresh and swollen Rhizopus oryzae spores and Aspergillus fumigatus conidia. Infect Immun 1985, 48:458-463 
30. Lewis RE, Albert ND, Kontoyiannis DP: Comparative pharmacodynamics of posaconazole in neutropenic murine models of invasive pulmonary aspergillosis and mucormycosis. Antimicrob Agents Chemother 2014, 58:6767-6772

31. Gao XD, Katsumoto T, Onodera K: Purification and characterization of chitin deacetylase from Absidia coerulea. J Biochem 1995, 117: $257-263$

32. Chamilos G, Ganguly D, Lande R, Gregorio J, Meller S, Goldman WE, Gilliet M, Kontoyiannis DP: Generation of IL-23 producing dendritic cells (DCs) by airborne fungi regulates fungal pathogenicity via the induction of $\mathrm{T}(\mathrm{H})-17$ responses. PLoS One 2010, 5:e12955

33. Lewis RE, Lortholary O, Spellberg B, Roilides E, Kontoyiannis DP, Walsh TJ: How does antifungal pharmacology differ for mucormycosis versus aspergillosis? Clin Infect Dis 2012, 54(Suppl 1): S67-S72

34. Gravelat FN, Beauvais A, Liu H, Lee MJ, Snarr BD, Chen D, Xu W, Kravtsov I, Hoareau CMQ, Vanier G, Urb M, Campoli P, Al Abdallah Q, Lehoux M, Chabot JC, Ouimet MC, Baptista SD, Fritz JH, Nierman WC, Latgé JP, Mitchell AP, Filler SG, Fontaine T, Sheppard DC: Aspergillus galactosaminogalactan mediates adherence to host constituents and conceals hyphal $\beta$-glucan from the immune system. PLoS Pathog 2013, 9:e1003575

35. Bouchara JP, Oumeziane NA, Lissitzky JC, Larcher G, Tronchin G, Chabasse D: Attachment of spores of the human pathogenic fungus Rhizopus oryzae to extracellular matrix components. Eur J Cell Biol 1996, 70:76-83

36. Bromley IM, Donaldson K: Binding of Aspergillus fumigatus spores to lung epithelial cells and basement membrane proteins: relevance to the asthmatic lung. Thorax 1996, 51:1203-1209

37. Liu M, Spellberg B, Phan QT, Fu Y, Fu Y, Lee AS, Edwards JE, Filler SG, Ibrahim AS: The endothelial cell receptor GRP78 is required for mucormycosis pathogenesis in diabetic mice. J Clin Invest 2010, 120:1914-1924

38. Chen Y, Nickola TJ, DiFronzo NL, Colberg-Poley AM, Rose MC: Dexamethasone-mediated repression of MUC5AC gene expression in human lung epithelial cells. Am J Respir Cell Mol Biol 2006, 34: 338-347

39. Kumar A, Shukla PK: A monoclonal antibody against glycoproteins of Aspergillus fumigatus shows anti-adhesive potential. Microb Pathog 2015, 79:24-30

40. Wiederhold NP, Kontoyiannis DP, Chi J, Prince RA, Tam VH, Lewis RE: Pharmacodynamics of caspofungin in a murine model of invasive pulmonary aspergillosis: evidence of concentrationdependent activity. J Infect Dis 2004, 190:1464-1471

41. Botterel F, Gross K, Ibrahim-Granet O, Khoufache K, Escabasse V, Coste A, Cordonnier C, Escudier E, Bretagne S: Phagocytosis of Aspergillus fumigatus conidia by primary nasal epithelial cells in vitro. BMC Microbiol 2008, 8:97

42. Wasylnka JA, Moore MM: Uptake of Aspergillus fumigatus conidia by phagocytic and nonphagocytic cells in vitro: quantitation using strains expressing green fluorescent protein. Infect Immun 2002, 70:3156-3163

43. Garlanda C, Hirsch E, Bozza S, Salustri A, De Acetis M, Nota R, Maccagno A, Riva F, Bottazzi B, Peri G, Doni A, Vago L, Botto M, De Santis R, Carminati P, Siracusa G, Altruda F, Vecchi A, Romani L, Mantovani A: Non-redundant role of the long pentraxin PTX3 in anti-fungal innate immune response. Nature 2002, 420: $182-186$

44. Kaur S, Gupta VK, Thiel S, Sarma PU, Madan T: Protective role of mannan-binding lectin in a murine model of invasive pulmonary aspergillosis. Clin Exp Immunol 2007, 148:382-389

45. Leleu C, Menotti J, Meneceur P, Choukri F, Sulahian A, Garin YJF, Derouin F: Efficacy of liposomal amphotericin B for prophylaxis of acute or reactivation models of invasive pulmonary aspergillosis. Mycoses 2013, 56:241-249

46. Bhatia S, Fei M, Yarlagadda M, Qi Z, Akira S, Saijo S, Iwakura Y, van Rooijen N, Gibson GA, St Croix CM, Ray A, Ray P: Rapid host defense against Aspergillus fumigatus involves alveolar macrophages with a predominance of alternatively activated phenotype. PLoS One 2011, 6:e15943

47. von Garnier C, Filgueira L, Wikstrom M, Smith M, Thomas JA, Strickland DH, Holt PG, Stumbles PA: Anatomical location determines the distribution and function of dendritic cells and other APCs in the respiratory tract. J Immunol 2005, 175:1609-1618

48. Fahy JV, Wong H, Liu J, Boushey HA: Comparison of samples collected by sputum induction and bronchoscopy from asthmatic and healthy subjects. Am J Respir Crit Care Med 1995, 152:53-58

49. Kopf M, Schneider C, Nobs SP: The development and function of lung-resident macrophages and dendritic cells. Nat Immunol 2015, $16: 36-44$

50. Philippe B, Ibrahim-Granet O, Prévost MC, Gougerot-Pocidalo MA, Sanchez Perez M, Van der Meeren A, Latgé JP: Killing of Aspergillus fumigatus by alveolar macrophages is mediated by reactive oxidant intermediates. Infect Immun 2003, 71:3034-3042

51. Sunahara KK, Martins JO: Alveolar macrophages in diabetes: friends or foes? J Leukoc Biol 2012, 91:871-876 